\title{
Three-boson system with absorptive short range potential
}

\author{
A. E. A. Amorim and Lauro Tomio \\ Instituto de Física Teórica, Universidade Estadual Paulista, 01405 São Paulo, São Paulo, Brazil \\ T. Frederico \\ Instituto de Estudos Avançados, Centro Técnico Aeroespacial, 12.231 São José dos Campos, São Paulo, Brazil
}

(Received 24 August 1992)

\begin{abstract}
We study the properties of the three-boson system with absorption, through a short range interaction in the limit where the range reduces to zero. We derive an analytic expression for the three-boson width that relates it to the real part of the three-boson energy, two-boson binding energy and decay constant. One of the characteristics of this expression is that, in this limit, the ratio between the width and the three-boson binding energy is proportional to the range.
\end{abstract}

PACS number(s): 25.10.+s, 21.45.+v, 25.43.+t

The formulation of three-body systems with absorption has application in many-body theory [1] and also in few-nucleon-antinucleon systems such as the antiproton deuteron [2-4]. The motivation for the study of the antiproton deuteron system relies on the possibility to test the nucleon-antinucleon interaction in the presence of a third nucleon. This gives the opportunity to check the much unknown imaginary part of the nucleonantinucleon potential under different conditions. The mechanism for the antinucleon annihilation on two nucleons [5] may also be studied.

The research in the antiproton deuteron atom is active $[3,6]$ and experimental data are available [7]. As a three-particle system, the energy and width of the antiproton deuteron system has been calculated [3] and also the spectator nucleon spectrum [4], using separable interaction model in Faddeev calculations.

In the three-nucleon system, qualitative studies have been done $[8,9]$ exploring the short range of the nuclear interaction. One of the intriguing characteristics of this system, first observed by Thomas [10], is the collapse of the three-body binding energy, when the interaction range of the two-body system goes to zero. This effect is also closely related to the appearance of the Efimov states $[8,9]$, as shown in Ref. [11], where it is demonstrated that both effects essentially arise from the same singularity structure of the kernel of the integral equation. Also related with Thomas and Efimov effects, and discussed in Ref. [11], is the model dependence of the three-particle observables.

Our main purpose is to discuss some general qualitative properties of a three-boson system with absorption through a schematic two-body potential model that has just what is essential to understand the behavior of the system in the limit where the range goes to zero. In the antiproton deuteron system the characteristic interaction distances are small compared to the deuteron size. To have an insight to the qualitative properties of such a system with absorption, we choose a two-body potential without any structure, but with the essential information about the range of the interaction. So, we use a range parameter that characterizes the interaction. When this parameter goes to infinity, the potential has a zero range. A potential without structure can be useful in this case to distinguish the main effects due to the range of the interaction from other effects. We also hope that such a study will be useful in understanding the limits one has to consider when using a more realistic two-body interaction. As in the case where there is no absorption, the system still collapses in the zero-range limit, but now the state has a width due to the imaginary part of the interaction.

The absorptive information present in the potential is supplied by the width of the two-boson state. Anticipating our results, the three-boson state has a width that, unlike the energy, does not increase as the inverse of the square of the interaction radius as it goes to zero. The width increases, but with the inverse of the interaction radius. Then, the collapsed state oscillates an infinite number of times before it decays.

We start presenting the zero range three-boson integral equation for the bound state [12]. We wrote it in a way that allows the input of two-boson data beyond the bound-state energy [13]:

$\chi(\mathbf{q})=2 \tau\left(E_{3}-\frac{3}{4} q^{2}\right) \int d^{3} k\left(E_{3}-q^{2}-k^{2}-\mathbf{q} \cdot \mathbf{k}\right)^{-1} \chi(\mathbf{k})$,

where $-E_{3}$ is the three-boson binding energy and we are using units such that $\hbar, c$, and the nucleon mass $m$ are equal to 1 . The two-boson $T$ matrix for the zero-range interaction is given by

$$
\tau(E)=\left[\lambda^{-1}-\int d^{3} p \frac{1}{E-p^{2}}\right]^{-1}
$$

The coupling constant $\lambda$ is fixed by one physical input, for example, the pole position of the two-boson scattering 
amplitude. The two-boson scattering amplitude has one pole, at $E_{2}$, which has in our case real and imaginary parts,

$$
\lambda^{-1}=\int d^{3} p \frac{1}{E_{2}-p^{2}} .
$$

The above value of $\lambda$, when substituted into Eq. (2), provides the cancellation of the linear divergence of the momentum integral and results [13]:

$$
\tau(E)=\left[2 \pi^{2}\left(\sqrt{-E_{2}}-\sqrt{-E}\right)\right]^{-1}
$$

A similar reasoning was applied in two spatial dimensions to determine the scattering amplitude for two particles [14]. The absorption in the zero range model is introduced by the width of the two-boson state, which is twice the imaginary part of $E_{2}$.

The $S$-wave projected three-boson integral equation, with the input of the two-boson energy, is given by

$$
\begin{aligned}
\chi(q)= & \frac{-2}{\pi\left[\sqrt{-E_{2}}-\sqrt{\frac{3}{4} q^{2}-E_{3}}\right]} \\
& \times \int_{0}^{\Lambda} d k \frac{k}{q} \ln \left[\frac{q^{2}+k^{2}+q k-E_{3}}{q^{2}+k^{2}-q k-E_{3}}\right] \chi(k),
\end{aligned}
$$

where the cutoff, $\Lambda$ goes to infinity as the radius of the interaction decreases. It qualitatively represents the inverse of the interaction radius [15]. The collapse of the three-boson binding energy is obtained for $\Lambda \rightarrow \infty$. In order to clarify our points, let us make a transformation of Eq. (5) to the units in which the cutoff is 1 . With rescaled variables $x \equiv k / \Lambda, y \equiv q / \Lambda$, and energies $\epsilon_{i} \equiv E_{i} / \Lambda^{2}$, we can redefine our projected $S$-wave such that

$$
f(y)=q \chi(q) \equiv \Lambda y \chi(\Lambda y)
$$

In this case we obtain

$$
\begin{aligned}
f(y)= & \frac{-2}{\pi\left[\sqrt{-\epsilon_{2}}-\sqrt{\frac{3}{4} y^{2}-\epsilon_{3}}\right]} \\
& \times \int_{0}^{1} d x \ln \left[\frac{y^{2}+x^{2}+y x-\epsilon_{3}}{y^{2}+x^{2}-y x-\epsilon_{3}}\right] f(x) .
\end{aligned}
$$

For fixed real $E_{2}$ the collapse of the three-boson energy comes from the existence of solutions of Eq. (7) in the limit $\epsilon_{2} \rightarrow 0$, where the inverse range parameter $\Lambda$ goes to infinity. In this limit, with $n$ labeling each bound state, $\epsilon_{3}^{n}$ are real and given by universal constants, as will be shown in our numerical results. The threeboson bound-state energies scale with the cutoff parameter, $E_{3}^{n}=\epsilon_{3}^{n} \Lambda^{2}$, and collapse in the limit $\Lambda \rightarrow \infty$. This is known as the Thomas effect [10]. The infinite number of states that appear in this limit are also known as the Efimov states [8], if one takes $\Lambda$ finite and constant as $E_{2}$ goes to zero. So, as it was also shown in Ref. [11], the Thomas and Efimov effects are directly related by a scale transformation, given by the inverse range parameter $\Lambda$ in our case.

The important point to be noted in Eq. (7) is that, for $E_{2}$ having an imaginary part, when the inverse range parameter goes to infinity, the spectrum of $\epsilon_{3}^{n}$ does not change. Thus, the first feature of the absorptive interaction in the zero range limit is that the real part of the energies of the three-boson states goes to infinity, and increases as the inverse of the square of the range parameter.

In order to calculate the width of the three-boson energies, we expand Eq. (7) in $\sqrt{-\epsilon_{2}}$. The ratio of $\epsilon_{2}$ to $\epsilon_{3}^{n}$ vanishes going to the zero range limit, due to the fixed value of $E_{2}$ and $\Lambda$ increases to infinite. Thus, we are allowed to perform such perturbative expansion. We introduce the perturbed quantities

$$
\epsilon_{3}^{\prime n}=\epsilon_{3}^{n}+\delta \epsilon_{3}^{n}
$$

and

$$
f^{\prime n}(y)=f^{n}(y)+\delta f^{n}(y) .
$$

The unperturbed energy and spectator function are calculated in the zero range limit. We observe that the prime quantities have real and imaginary parts, when the two-body bound state decays. Corresponding to the case $\epsilon_{2}=0$, the quantities $\epsilon_{3}^{n}$ and $f^{n}(y)$ are real, as one can obviously see from Eq. (7). In the next we use a first order correction in $\sqrt{-\epsilon_{2}}$ for the $\delta$ quantities.

The expansion of Eq. (7) in lowest order in $\sqrt{-\epsilon_{2}}$, where we make use of the unperturbed equation to simplify the expression, is written below

$$
\begin{aligned}
\delta f^{n}(y)= & \left(\sqrt{-\epsilon_{2}}+\frac{\delta \epsilon_{3}^{n}}{2 \sqrt{\frac{3}{4} y^{2}-\epsilon_{3}^{n}}}\right) \frac{f^{n}(y)}{\sqrt{\frac{3}{4} y^{2}-\epsilon_{3}^{n}}} \\
& +\frac{2 / \pi}{\sqrt{\frac{3}{4} y^{2}-\epsilon_{3}^{n}}} \int_{0}^{1} d x\left[\ln \left(\frac{y^{2}+x^{2}+y x-\epsilon_{3}^{n}}{y^{2}+x^{2}-y x-\epsilon_{3}^{n}}\right) \delta f^{n}(x)+\frac{2 y x f^{n}(x) \delta \epsilon_{3}^{n}}{\left(y^{2}+x^{2}-\epsilon_{3}^{n}\right)^{2}-(y x)^{2}}\right] .
\end{aligned}
$$

From the linearity of the above equation, the change in the three-boson energy is given by

$$
\delta \epsilon_{3}^{n}=-C_{n} \sqrt{-\epsilon_{2}},
$$

where $C_{n}$ are the coefficients that come from the solution of Eq. (10) for each three-boson state. These coefficients attain universal values depending only on the structure of the three-boson integral equation, Eq. (7). They are independent of the two-boson energy.

The coefficients $C_{n}$ are also approximately propor- 
tional to $\sqrt{-\epsilon_{3}^{n}}$, such that

$$
\lim _{n \rightarrow \infty} \frac{C_{n}}{\sqrt{-\epsilon_{3}^{n}}}=\alpha
$$

where $\alpha$ is a well-defined constant that will be obtained numerically. To qualitatively understand this property, we must consider that $-\epsilon_{3}^{n}<<1$, as shown in Table I. The value of $\epsilon_{3}^{n}$ in Eq. (10) is important only when $y^{2} \approx-\epsilon_{3}^{n}$. The inhomogeneous term in Eq. (10), i.e., the first term in the right-hand side (RHS), increases as the inverse of $\sqrt{-\epsilon_{3}^{n}}$ for $-\epsilon_{3}^{n}$ going to zero. In the second term in the RHS of Eq. (10), $\delta \epsilon_{3}^{n}$ must be proportional to $\sqrt{-\epsilon_{3}^{n}}$ to produce the same behavior as the inhomogeneous term when $-\epsilon_{3}^{n}$ vanishes, as required by the linearity of such equation. Thus, roughly we have

$$
\delta \epsilon_{3}^{n} \sim-2 \sqrt{-\epsilon_{3}^{n}} \sqrt{-\epsilon_{2}} .
$$

We do not consider the behavior of $f^{n}$, since the normalization is free and we always can rescale it. These considerations are substantiated by our numerical results presented in Table I.

We give in Table I the first few unperturbed energies, the coefficients $C_{n}$ and the ratios $C_{n} / \sqrt{-\epsilon_{3}^{n}}$ for the threeboson bound states. One may observe the constancy of these ratios for a wide range of variation in the magnitude of $\epsilon_{3}^{n}$ and $C_{n}$, where we found $\alpha$, given by Eq. (12), to be very close to 2 , as also anticipated by our above discussion. It is worthwhile to mention that the adimensional quantities $\epsilon_{3}^{n}$ are in fact the energies of the Efimov states for an interaction with a finite range of $\Lambda^{-1}=1$ and zero two-body bound-state energies. The collapsed energies of the Thomas states [8] are obtained in the units where $\Lambda^{-1}$ goes to zero and the two-body bound state is finite. This numerically illustrates the connection between Thomas and Efimov states [11].
TABLE I. First few energies and universal coefficients $C_{n}$ of the collapsed three-boson states. In the last column is presented the ratio $C_{n} / \sqrt{-\epsilon_{3}^{n}}$.

\begin{tabular}{lllc}
\hline \hline $\mathbf{n}$ & $-\epsilon_{3}^{n}$ & $C_{n}$ & $C_{n} / \sqrt{-\epsilon_{3}^{n}}$ \\
\hline 0 & $3.166 \times 10^{-2}$ & $3.570 \times 10^{-1}$ & 2.0 \\
1 & $6.021 \times 10^{-5}$ & $1.640 \times 10^{-2}$ & 2.1 \\
2 & $1.174 \times 10^{-7}$ & $7.193 \times 10^{-4}$ & 2.1 \\
\hline \hline
\end{tabular}

We present below the three-boson width as a function of the observables of the system. The real part of $E_{3}^{n}$ gives us the inverse range of the force, $\Lambda$, which is used to obtain the width of the three boson. The expression for the inverse range parameter, as well as the expression for the three-boson width, are obtained from the real and imaginary parts of $E_{3}^{n}$, using Eq. (11):

$$
\begin{aligned}
E_{3}^{n}=\left(\epsilon_{3}^{n}+\delta \epsilon_{3}^{n}\right) \Lambda^{2}=\left(\epsilon_{3}^{n}-C_{n} \sqrt{-\epsilon_{2}}\right) \Lambda^{2} \\
=\epsilon_{3}^{n} \Lambda^{2}-C_{n} \sqrt{-E_{2} \Lambda} \\
=\epsilon_{3}^{n} \Lambda^{2}-C_{n}\left[\sqrt{\frac{\left|E_{2}\right|-\operatorname{Re}\left(E_{2}\right)}{2}}\right. \\
\left.+i \sqrt{\frac{\left|E_{2}\right|+\operatorname{Re}\left(E_{2}\right)}{2}}\right] \Lambda,
\end{aligned}
$$

where $\left|E_{2}\right|=\sqrt{\left[\operatorname{Re}\left(E_{2}\right)\right]^{2}+\left(\Gamma_{2} / 2\right)^{2}}$ and $\Gamma_{2}$ is the twoboson width.

From Eq. (14), the width of the collapsed states, $\Gamma_{3}^{n}$, are given by

$$
\Gamma_{3}^{n}=2 C_{n} \sqrt{\frac{\left|E_{2}\right|+\operatorname{Re}\left(E_{2}\right)}{2}} \Lambda,
$$

and, from the real part of Eq.(14), we have the inverse range parameter:

$$
\begin{aligned}
\Lambda=\left(\frac{1}{2 \sqrt{-\epsilon_{3}^{n}}}\right)\left[\frac{C_{n}}{-\sqrt{-\epsilon_{3}^{n}}} \sqrt{\frac{\left|E_{2}\right|-\operatorname{Re}\left(E_{2}\right)}{2}}\right. \\
+\sqrt{\left.\left(\frac{C_{n}}{\sqrt{-\epsilon_{3}}}\right)^{2}\left(\frac{\left|E_{2}\right|-\operatorname{Re}\left(E_{2}\right)}{2}\right)-4 R e\left(E_{3}^{n}\right)\right]}
\end{aligned}
$$

The width increases linearly in contrast with the real part of the energy that increases quadratically with the inverse range of the interaction.

Finally, by replacing $\Lambda$, given by Eq. (16), in Eq. (15), we obtain the expression that correlates the three-boson width with the real part of the three-boson energy, as a function of the two-boson width and energy:

$$
\Gamma_{3} \cong\left(\frac{\alpha}{2}\right)^{2} \Gamma_{2}\left[\sqrt{1+\left(\frac{2}{\alpha}\right)^{2} \frac{2 \operatorname{Re}\left(-E_{3}\right)}{\left|E_{2}\right|-\operatorname{Re}\left(E_{2}\right)}}-1\right]
$$

Following our previous argumentation we drop the label $n$, as $C_{n} / \sqrt{-\epsilon_{3}^{n}}$ is approximately constant, as given by Eq. (12) and shown in Table I. We should observe that the analytical expressions written above are valid only in the limit of zero interaction range, but we believe that can be very useful in understanding the limits one has to consider in a more realistic situation.

In Fig. 1 we present a plot showing the correlation between the width $\Gamma_{3}$ and the real part of the three-boson energy. The set of curves presented are given for different values of the two-body energy ratio $\Gamma_{2} /\left(2\left|E_{2}\right|\right)$. All quantities are shown in units of the absolute value of the 
two-boson energy and for $\alpha=2$. As one can see in this plot, the values of $\Gamma_{3}$ as a function of $\operatorname{Re}\left(E_{3}\right)$ have an upper limit given by the curve that has $\left|E_{2}\right|=\Gamma_{2} / 2$.

In summary, we show that the absorption in the twoboson interaction does not prevent the collapse of the three-boson system in the zero range limit. The width of the collapsed states increases linearly with the inverse of the range of the interaction. It is negligible compared to the real part of the three-boson energy in this limit. We obtain numerically the universal proportionality coefficients for the variation of the three-boson energy with the square root of the two-boson energy. It is important to mention that this schematic model has only the physical information about the two-boson pole in the scattering amplitude. For practical applications, with short ranged potentials, one may note that the physical information contained in the two-body scattering amplitude goes behyond the two-boson pole, and this may reflect in a different value of the $\alpha$ coefficient. We expect that in such a case the general behavior of the imaginary part of the three-boson energy will be correlated with the real part in a similar way, as shown in Fig. 1.

We have presented, in a schematic model, some simple explanations of general features of a three-boson system with absorption that as far as we know have not been pointed out before. Our conclusions can shed light to the physics of a more realistic three-boson system with absorption. With a realistic local interaction a correspondent analytical study is not so easy to be followed, and we think that may be unnecessary, because the main characteristic of such a system, relevant for the present study, is already presented in our schematic approach. A study of the model dependence of the three-boson observables with general short range absorptive potentials can easily be followed by using separable interactions.

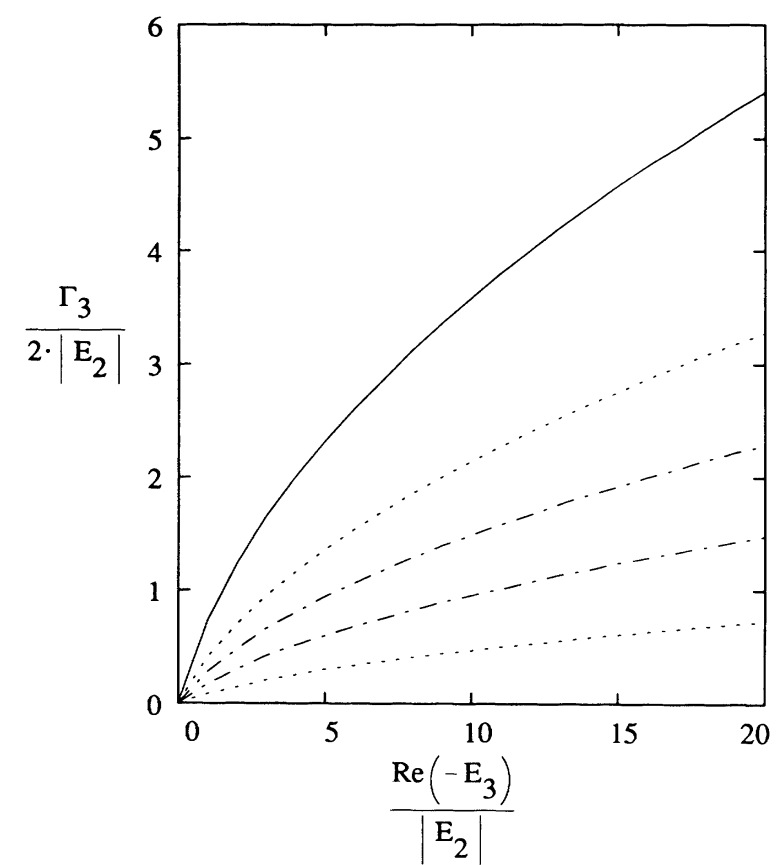

FIG. 1. Plot of the imaginary part against the real part of the three-body energy, given by Eq. (17). All quantities are given in units of the absolute value of the complex two-body energy, and for the $\alpha$ coefficient equal to 2 . The set of curves presented are given for $\Gamma_{2} /\left(2\left|E_{2}\right|\right)=0.2,0.4,0.6,0.8$, and 1.0 , where the solid curve, $\Gamma_{2} /\left(2\left|E_{2}\right|\right)=1$, shows the upper limit for $\Gamma_{3} /\left(2\left|E_{2}\right|\right)$.

We are grateful to Prof. S. K. Adhikari for relevant comments. This work was supported by the Conselho Nacional de Desenvolvimento Científico e Tecnológico CNPq.
[1] N. Austern, Y. Iseri, M. Kamimura, M. Kawai, G. Rawitscher, and M. Yahiro, Phys. Rep. 154, 125 (1987).

[2] M. Zh. Shmatikov and I.L. Grach, Yad. Fiz. 34, 967 (1981) [Sov. J. Nucl. Phys. 34, 538 (1981)].

[3] G.P. Latta and P.C. Tandy, Phys. Lett. B 209, 14 (1988); Phys. Rev. C 42, 1207 (1990).

[4] T. Frederico, B.V. Carlson, R.C. Mastroleo, L. Tomio, and M.S. Hussein, Phys. Rev. C 42, 138 (1990).

[5] J. Cugnon and J. Vandermeulen, Phys. Lett. 146B, 16 (1984).

[6] G.Q. Liu, L.M. Richard, and S. Wyceck, Phys. Lett. B 260, 15 (1991).

[7] C. Amsler and F. Myhrer, Annu. Rev. Nucl. Part. Sci. 41, 219 (1991); C.J. Batty, Rep. Prog. Phys. 52, 1165 (1989).

[8] V. Efimov, Phys. Lett. 33B, 563 (1970); V. Efimov and E. A. Tkachenko, Few Body Syst. 4, 71 (1988); V. Efimov, Commun. Nucl. Part. Phys. 19, 271 (1990).
[9] L. Tomio, T. Frederico, and A. Delfino, Phys. Rev. C 41, 876 (1990); see also S. K. Adhikari and K. L. Kowalski, Dynamical Collision Theory and Its Applications (Academic, Boston, 1991), Chap. 7.

[10] L.H. Thomas, Phys. Rev. 47, 903 (1935).

[11] S.K. Adhikari, A. Delfino, T. Frederico, I.D. Goldman, and L. Tomio, Phys. Rev. A 37, 3666 (1988).

[12] G.V. Skornyakov and K.A. Ter-Martirosian, Zh. Eksp. Teor. Fiz. 31, 775 (1957) [Sov. Phys. JETP 4, 648 (1957)].

[13] T. Frederico, I.D. Goldman, and A. Delfino, Phys. Rev. C 37, 497 (1988).

[14] R. Jackiw, in Breaking of Classical Symmetries by Quantum Effects, Symposium on Quantum Physics, Geneva, Switzerland, 1991 (MIT Report No. MIT-CTP 1971).

[15] V.F. Kharchenko, Yad. Fiz. 16, 310 (1972) [Sov. J. Nucl. Phys. 16, 173 (1973)]. 